\title{
Vegetationsgeschichtliche Untersuchungen an altpleistozänen Ablagerungen aus Lieth bei Elmshorn
}

\author{
Von Burchard Menke, Kiel \\ Mit 1 Abbildung und 4 Tafeln
}

$\mathrm{Z}$ us a m men fassung. In der "Meinert'schen Kalkgrube“ in Lieth bei Elmshorn (Westholstein) wurde durch pollenanalytische Untersuchungen an organogenen Ablagerungen, die hier in einer Karstsenke liegen, eine altpleistozäne, vor-cromerzeitliche Schichtfolge nachgewiesen. Ihre Vegetationsentwicklung läßt eine Reihe von Klimaschwankungen erkennen. Die Möglichkeit ihrer Eingliederung in die bisherige Altpleistozän-Stratigraphie wird diskutiert.

$\mathrm{Summary}$. Organic layers, situated in a sink whole of the surface of the Zechstein, digged off in the "Meinert Lime Pit" in Lieth (Elmshorn, Schleswig-Holstein - NW Germany), were deposited during a Pre-Cromerian period of the Early-Pleistocene. By means of pollenanalytical intestigations several oscillations of the climate were discovered. It is a matter of discussion, in how far they fit in the development of the Early Pleistocene, known up to now.

\section{Einleitung}

Seit Jahrzehnten beansprucht die „Meiert'sche Kalkgrube“ in Lieth bei Elmshorn großes erdgeschichtliches Interesse (GRIPP 1964, DüCKER \& MEnke 1968). Abgebaut wird hier der Zechsteinkalk. Seine Oberfläche ist von Verkarstungserscheinungen gekennzeichnet. Die Karstsenken haben als „Sedimentfallen“ eine besondere Bedeutung. Ihre Füllungen bestehen aus Sanden und organogenen Ablagerungen, die hier vor Abrasion geschützt waren. Neue Aufschlüsse aus den Jahren 1966/67 und 1968 bieten die Möglichkeit einer erneuten vegetationsgeschichtlichen Bearbeitung derartiger organogener Ablagerungen (Dǘcker \& Menke 1968, Lüttig, Menke \& Schneekloth 1967). Besonders herzlich danken wir den Gebrüdern HeLl, Inhaber der Liether Kalkwerke, für ihre Erlaubnis und ihr Entgegenkommen.

\section{Lagerung und Petrographie}

Die organogenen Ablagerungen bestehen aus extrem gepreßten Torfen und Mudden, die eine braunkohleartige Konsistenz haben. Es sind mehrere Flöze vorhanden, die durch Sande und (in geringerem Maße) Schluffe voneinander getrennt sind (Abb. 1). Der neue Aufschluß liegt an der Südflanke einer Karstsenke. Die Schichten fallen mit durchschnittlich etwa $40-60^{\circ}$ zur Senke hin ein, sie streichen in Ost-West-(bis ESE-NNW)-Richtung. Die Flöze sind stark zerklüftet, besonders starke Störungen weist der Komplex „C“ (Abb. 1) auf. Die oberflächennahen Schichten der Serie sind jeweils mehr oder minder stark „verbrodelt“. Darüber liegen Fließerden, die mit einer windkanterführenden Steinsohle abschließen. Dann folgen geschichtete „Decksande“, in die drei humose Horizonte eingelagert sind, deren oberer durch eine etwa $4 \mathrm{~cm}$ mächtige torfige Humuslage und eine deutliche Bleicherde gekennzeichnet ist (wahrscheinlich Alleröd-Interstadial).

Für die pollenanalytischen Untersuchungen wurden sorgfältig solche Profile ausgewählt, die keine Lagerungsstörungen erkennen ließen; trotzdem ist im Profil „Bremsberg“ (Tafel I) offenbar eine solche vorhanden.

\section{Darstellung}

Die Darstellung der Pollendiagramme (Tafel I bis IV) erfolgte nach dem „IVERSEN-Verfahren“ mit der Gesamtsumme der Pollen (mit Ausnahme der Wasserpflanzen- und Cupressaceae/Taxodiaceae-Pollen) als Bezugsbasis ("Grundsumme"). Die Sporen und Wasserpflanzen-Pollen wurden auf die Grundsumme bezogen, die Cupressaceae/Taxodiaceae- (incl. "Juniperus-Typ“) Pollen auf 


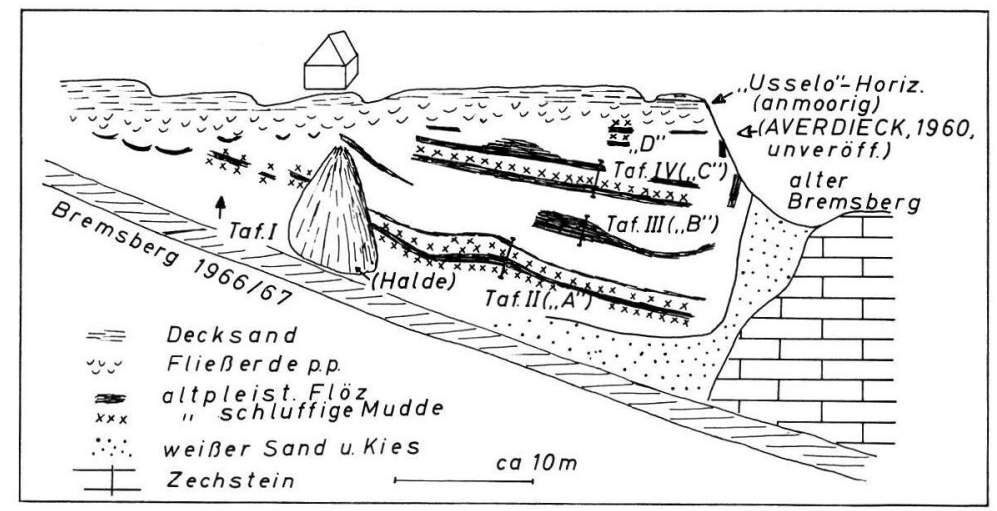

Abb. 1. Lagerungsverhältnisse im Aufschluß 1968 am Bremsberg der Meinert'schen Kalkgrube in Lieth nach einer Faustskizze. Blick nach Norden.

die um diese Typen vermehrte Grundsumme. Die Summe der „Bäume und Sträucher“ (im folgenden kurz als „Baumpollen“ bezeichnet) schließt Corylus, Myrica, Salix, Alnus cf. viridis, FrangulaTyp, Ilex, "Rhamnaceae-Typ“ u. a. ein. Der Pollen vom „Rhamnaceae-Typ“ konnte allerdings noch nicht bestimmt werden; wahrscheinlich handelt es sich nicht um eine Rhamnacee. Eine Beschreibung und Diskussion der Pollentypen ist an dieser Stelle aus räumlichen Gründen nicht möglich. Sie wird später nachgeholt werden. Die Pollenerhaltung war im allgemeinen sehr gut.

\section{Vegetationsentwicklung}

\subsection{Begriff sbestim mung}

Die Pollendiagramme lassen auf eine Anzahl von Klimaschwankungen schließen. Von einer „W a r m z e i t“ wird im folgenden gesprochen, wenn eine „vollständige“, d. h. mit einer thermophilen Gehölzflora kulminierende Waldentwicklung nachweisbar ist. Die Grenze einer „Kalt ze i t" zur Warmzeit wird an den Beginn bzw. das Ende einer "subarktischen Parklandschaft“ (ZAGwiJN 1957, 1960) gelegt. Schwierig wird die Entscheidung ob eine Kaltzeit vorliegt oder nicht, wenn die Entwicklung von einer thermophilen Gehölzflora nicht bis zu einer eindeutig „subarktischen Parklandschaft" führt.

Wenn die Vegetationsentwicklung während einer W a rmzeit auf eine zeitweilige, merkliche Abkühlung schließen läßt, ohne daß diese zu einer "subarktischen Parklandschaft" führt, so wird der "kühle" Abschnitt im folgenden als "K r y o phas e“, die sie flankierenden "warmen" Abschnitte werden als „T h e r m o ph a s e n“ bezeichnet.

Führt die Entwicklung in „warmen“ Abschnitten innerhalb einer $\mathrm{Kaltze}$ it bis höchstens zu „borealen“ Waldtypen, so werden diese Abschnitte als „In te r s t a dia l“, der vorangehende bzw. folgende „kalte“ Abschnitt wird als „S t a d i a l" bezeichnet. Die Abgrenzung eines Interstadials zur Warmzeit wird unsicher, wenn in geringem Maße eine thermophile Gehölzflora auftritt. Es ist zu berücksichtigen, daß thermophile Gehölze (z. B. Ulmus, Quercus) heute weit in die borealen Regionen vordringen. Außerdem müssen die heutigen Vegetationszonierungen und Arealgrenzen nicht unbedingt auch als Modelle für die früheren Verhältnisse brauchbar sein.

Diese Definitionen können natürlich nur für ein bestimmtes Gebiet gelten; wenn z. B. die Waldentwicklung während eines bestimmten Zeitabschnittes in Schleswig-Holstein bis zu einer "subarktischen Parklandschaft" führte, so muß dies natürlich nicht auch für ganz Mitteleuropa zutreffen. Damit werden aber auch Konnektierungen über größere Gebiete hinweg u. U. schwierig sein. Zur Zeit kann daher nur versucht werden, die Entwicklung zunächst jeweils ganz lokal so weit wie möglich zu rekonstruieren.

\subsection{Ekholt $-\mathrm{Kaltze}$ it}

An der Basis des Flözes „A“ (Abb. 1, Tafel I und II) liegt eine schluffige, gelbbraune Mudde. Das NBP/BP-Verhältnis ist hier sehr hoch (Zone E). Der Baumpollen erreicht jeweils nur geringe Anteile. Pollen thermophiler Taxa ist nur in Spuren vorhanden, die sich auf sekundärer Lagerstätte befinden dürften, wie auch der Pollen typischer Tertiär- 
gehölze, der - wie in allen stark mineralhaltigen Ablagerungen der Serie - ebenfalls in Spuren vorhanden ist. Unter den „Kräutern“ sind vor allem Artemisia, Thalictrum, Rubiaceae und Rosaceae zu nennen; häufig wurden auch Sporen vom Selaginella selaginoides-Typ gefunden. Im übrigen dominiert der Gramineen- und Cyperaceen-Pollen, während die Ericales-Anteile noch gering sind.

Die Pollenspektren sind zweifellos als Ausdruck einer offenen, kaltzeitlichen Vegetation zu werten. Der Verlauf der weiteren Entwicklung spricht ebenfalls klar für diese Deutung. Diese Kaltzeit wurde als „Ekholt“-Kaltzeit bezeichnet (Dücker \& Menke 1968).

Die Vegetation enthielt — so weit die Pollenanalysen darüber Auskunft geben - im Gegensatz zu den bisher in Schleswig-Holstein näher untersuchten Floren des Saale- und Weichsel-Spätglazials - anscheinend kaum typisch basiphile Heliophyten. Möglicherweise herrschten mehr oder minder oligotrophe Bedingungen.

\subsection{Nordende-Warmzeit}

An der Grenze zwischen der schluffigen Mudde und den mineralarmen, organogenen Ablagerungen (Mudde) beginnt die „Nordende“-Warmzeit (Dücker \& Menke 1968) mit einem markanten Abfall des NBP/BP-Verhältnisses und der Salix-Anteile, sowie einer Zunahme der Betula-Anteile als Ausdruck der einsetzenden Bewaldung (Tafel I und II). Die „B irken-Z e i t“ $\left(\mathrm{N}_{1}\right)$ ist nur schwach ausgeprägt. Mit ihrem Beginn nehmen die Pollenanteile vom Alnus viridis-Typ (der sich gut vom Alnus glutinosa-Typ abtrennen läßt) deutlich zu.

Die heutige Alnus viridis ist eine strauchförmige Erle, die in zahlreichen Sippen (die heute als selbständige Arten aufgefaßt werden) in den borealen Gebieten (und in den montan-subalpinen Regionen der Gebirge) Eurasiens (außer Nordeuropa) und Nordamerikas verbreitet ist. Die Grünerle bevorzugt feuchte Standorte auf kalkarmen, mäßig sauren Böden. In den Alpen tritt sie bestandbildend vor allem in kühl-humider Klimalage im subalpinen Knieholzgürtel auf, während sie in tieferen Lagen vor allem in Schneerunsen und an Bachrändern zu finden ist (OBERDORFER 1962).

Während der „K i e f e r n-Z e i t“ $\left(\mathrm{N}_{2}\right)$ setzt sich der Abfall des NBP/BP-Verhältnisses fort. Alnus cf. viridis kulminiert mit Anteilen um 3-4\%. Von Anfang an ist der Pollen thermophiler Taxa vorhanden: Zuerst erscheint Ulmus, dann Quercus, später der Pollen vom Alnus glutinosa-Typ. Im Laufe des Abschnittes breitet sich Osmunda aus, eine thermophile Farngattung, die saure Humusböden bevorzugt. Mit dem Beginn der "Ersten Mischwald-Zeit“ $\left(\mathrm{N}_{3}\right)$ setzt etwa gleichzeitig die Hauptausbreitung von Alnus of glutinosa, cf Ostrya (auch Ostryopsis und Carpinus-Arten können in Betracht kommen) und Ulmus sowie der Beginn der Ausbreitung von Carpinus ein. Zuerst kulminieren Ulmus und of Ostrya, dann erreicht Quercus eine starke Dominanz. Die Pinus-Anteile sinken auf geringe Werte, so daß die Kiefer als Waldbildner wohl keine nennenswerte Rolle mehr spielte. In den Spektren der Ersten Mischwald-Zeit drücken sich Waldtypen eines gemäßigten Klimas aus, in denen Ulme, Eiche, Hopfenbuche (oder Verwandte) eine große Rolle spielten, die Hainbuche sich ausbreitete, während Kiefer, Fichte, Eibe, Hasel wohl nur eine sehr untergeordnete Bedeutung hatten, Tanne und Linde aber anscheinend fehlten. Bezeichnend ist der allmähliche Rückgang des Pollens vom Alnus viridis-Typ. Es folgt eine " $\mathrm{Z}_{\mathrm{w}}$ e it e $\mathrm{M}$ ischwald-Zeit" $\left(\mathrm{N}_{4}\right)$, die durch höhere Carpinus- und geringere Quercus- und cf Ostrya-Anteile, vor allem jedoch durch die Ausbreitung von Eucommia und Pterocarya gekennzeichnet ist. In diesem Abschnitt ist der cf Cupressaceae-Pollen ("Juniperus-Typ“) etwas häufiger; es kann sich um Pollen von Tertiär-Relikten handeln. Pollen weiterer Tertiär-Relikte tritt in Spuren auf (cf Celtis, Symplocos, cf Cletbra u. a.). Sie befinden sich wohl auf primärer Lagerstätte, können aber fernverbreitet sein. Erwähnenswert sind zahlreiche Pollenfunde von Ilex, während Myrica-Pollen praktisch nicht vorhanden ist. 


\subsection{K r ü ckau-Kaltze it}

Die Entwicklung bricht mit einer geringmächtigen Sandschicht ab (Tafel II). Darüber liegt wieder eine sandig-schluffige Mudde $\left(K_{0}\right)$ mit ähnlichen Kriterien wie im Abschnitt E, jedoch mit erheblicher Ericales-, Myrica- und Alnus of viridis-Beteiligung. Diese Unterschiede schließen - wie auch die Lagerungsverhältnisse - eine Verdoppelung der unteren Serie aus, so daß es sich hier um eine jüngere Kaltzeit handelt. Zu erwähnen sind Sporenfunde vom Selaginella selaginoides- und Selaginella belvetica-Typ, von Lycopodium selago und wiederum höhere Anteile von Artemisia und Thalictrum. Die Umgebung war also offenbar eine ozeanisch-subarktische Landschaft mit Gebüschen und Heiden. Pollen und Sporen thermophiler Taxa, die in Spuren auftreten, befinden sich wohl auf sekundärer Lagerstätte. Mit dem Abschnitt $\mathrm{K}_{\mathrm{o}}$ beginnt somit eine Kaltzeit, die als „Krückau“-Kaltzeit bezeichnet wurde (DüCKER \& Menke 1968).

Dann folgt erneut eine geringmächtige, organogene Serie mit einer „Birken-Zeit“ $\left(K_{1}\right)$, einer "Kiefern-Zeit“ $\left(\mathrm{K}_{2}\right)$, in der Ulmus und Quercus wieder geschlossene Kurven erreichen, und einer beginnenden "Mischwald-Zeit" $\left(\mathrm{K}_{3}\right)$, in der sich Alnus cf glutinosa, Quercus, Ulmus, Carpinus und Osmunda erneut kurzfristig ausbreiten. Pinus, Alnus cf viridis und Myrica bleiben in diesen Abschnitten gut vertreten. Die Entwicklung wird dann offenbar erneut durch einen Rückschlag $\left(\mathrm{K}_{4}\right)$ abgebrochen, demzufolge die thermophilen Elemente wieder verschwinden. In den Abschnitten $\mathrm{K}_{3}$ und $\mathrm{K}_{4}$ breiten sich Alnus cf viridis und vor allem Ericales stark aus. Es scheint jetzt auch Larix vorhanden gewesen zu sein. Läßt man mit dem Abschnitt $\mathrm{K}_{0}$ eine selbständige Kaltzeit beginnen, wie es hier aufgrund der oben gegebenen Definition geschehen ist, so sind die Abschnitte $\mathrm{K}_{1}$ bis $\mathrm{K}_{3}$ als ein Interstadial anzusehen, der Abschnitt $\mathrm{K}_{4}$ als ein Stadial.

Zwischen den Flözen „A“ und „B“ (Abb. 1) liegt eine Sandschicht von wechselnder (in diesem Fall 1,60 m) Mächtigkeit. Sie kann wohl mit dem für den Abschnitt $\mathrm{K}_{4}$ wahrscheinlich gemachten Klima-Rückschlag in Beziehung gebracht werden.

Das Flöz „B“ (Tafel III) ist auf diesem Sand allem Anschein nach natürlich gewachsen. Petrographisch besteht es hauptsächlich aus Ablagerungen eines Ericales-SphagnumMoores. In diesem Zusammenhang sind ein Pollenfund von Drosera, Sporenfunde vom Lycopodium inundatum-Typ und hohe Ericales-Anteile zu nennen. Das braunkohlenartige Material enthält Sphagnum-Reste, Tilletia-Sporen und z. T. reichlich Pilzhyphen, wie dies ähnlich auch von holozänen Ablagerungen aus Heide- und trockneren Hochmooren geläufig ist. Gelegentlich gefundene of Pinus-Tracheiden lassen Lokalvorkommen von Pinus vermuten. Größere Hölzer sind allerdings selten. Weiterhin ist vor allem im älteren Teil mit gelegentlichen Lokalvorkommen von Alnus of viridis und Myrica zu rechnen.

Unter den Baumpollen (Tafel III) herrschen Pinus und Betula. Erheblich vertreten sind außer den Ericales (darunter Calluna) weiterhin vor allem Alnus cf viridis, Myrica und Cupressaceae-Typen. Pollen vom Larix-Typ, der anscheinend vor allem in „borealen" Spektrentypen auftritt, ist ebenfalls nicht selten. Andererseits erreichen aber auch Pollen und Sporen thermophiler Taxa nicht unerhebliche Anteile; vor allem sind zu nennen: Alnus cf glutinosa, Ulmus, Quercus, Carpinus und Osmunda. Ihre Anteile überschreiten aber (mit Ausnahme von Alnus of glutinosa) in keiner Probe 5\% der Pollensumme. Die Carpinus-Anteile bleiben im Durchschnitt sogar unter 1\%. Der Pollen vom OstryaTyp und von Corylus erreicht mehr oder minder geschlossene Kurven, jedoch kann er fernverbreitet sein. Eucommia-Pollen tritt vor allem im jüngeren Teil des Abschnittes $\mathrm{K}_{5}$ mit geringen Anteilen auf, während Pollenfunde von Pterocarya auf zwei Proben beschränkt bleiben. Beide Taxa müssen nicht in der engeren Umgebung vorgekommen sein.

Die Deutung der Spektren ist nicht einfach: Der Umgebungsniederschlag wird durch den zweifellos erheblichen Lokalniederschlag relativ gedrückt, wobei sich das Ausmaß freilich schwer beurteilen läßt. Trotzdem sprechen die Spektren für mehr oder minder 
boreal-subboreale Verhältnisse. Das wird besonders deutlich, wenn man das Flöz „C“ (Tafel IV) zum Vergleich heranzieht, in dem zweimal ganz ähnliche Lokalverhältnisse herrschten. Der mächtige Torf des Flözes „B“ spricht nicht gegen mehr oder minder "boreale" Verhältnisse: Heide- und Sphagnum-Moore sind sowohl in ozeanisch-gemäßigten als auch in borealen Zonen verbreitet.

Thermophile Elemente können vor allem unter eu-ozeanischen Bedingungen mehr oder minder weit in die borealen Gebiete vordringen. Erinnert sei in dem Zusammenhang auch an das heutige Vorkommen mediterraner Arten in England und Irland.

Es läßt sich insgesamt wohl die Auffassung vertreten, der Abschnitt $\mathrm{K}_{5}$ (Tafel III) sei als ein großes Interstadial der Krückau-Kaltzeit anzusehen; es wird vorläufig als „E11 e r hoo p - I n t e r st a d i a l“ bezeichnet.

Andererseits muß aber auch zur Diskussion gestellt werden, ob die im ganzen geringen Anteile thermophiler Taxa lediglich durch eine lokale Überrepräsentanz vor allem des Ericales- und Pinus-Pollens verursacht sein können. In diesem Fall ließe sich das Flöz „B“ als eine warmzeitliche Ablagerung auffassen.

Man könnte dazu u. a. auf die häufigen Pollenfunde von Eucommia verweisen. Die heutige, in Ostasien beheimatete Eucommia ulmoides ist eine ausgesprochen thermophile Art (WANG 1961). Damit wissen wir über die Ansprüche der im späten Tertiär bei uns heimischen „Eucommia europaea" (MäDLER, zit. ZaGWIJN 1960), um die es sich wohl im vorliegenden Fall handelt, allerdings noch nichts.

Eine eindeutige Entscheidung, welche von beiden Möglichkeiten der Deutung zutrifft, ist zur Zeit noch nicht möglich. Im Abschnitt K6 herrschten dagegen zweifellos „boreale“ Verhältnisse; das Verschwinden der thermophilen Elemente ist eindeutig belegt. Abgesehen von einer Zunahme der Alnus cf viridis-, Cyperaceen- und Gramineen-Anteile sind die Veränderungen im übrigen gering. Später $\left(\mathrm{K}_{7}\right)$ wird das Moor allmählich mit Sand überdeckt. Im Verein mit dem im Abschnitt K6 nachgewiesenen Klima-Rückschlag läßt sich diese Sandschicht ursächlich mit arktisch-subarktischen Verhältnissen in Beziehung bringen. Sieht man den Abschnitt $\mathrm{K}_{5}$ als ein Groß-Interstadial der Krückau-Kaltzeit an, wie es hier geschehen ist, so gehört der hangende Sand des Flözes „B“ dieser Kaltzeit als stadiale Ablagerung an.

\subsection{Tornesch-W a r m zeit}

Das Flöz „C“ (Abb. 1, Tafel IV) ist auf dem hangenden Sand des Flözes „B“ offenbar natürlich gewachsen. Das Flöz setzt mit einem Torf ein, der überwiegend in einem Ericales-Sphagnum-Moor gewachsen ist. Dann folgt eine tonig-schluffige, sandstreifige Mudde; das Moor wurde also überflutet. Diese Mudde wird von einer mineralarmen Mudde überlagert. Die limnischen Ablagerungen wurden dann übersandet (ca. $40 \mathrm{~cm}$ Sand). Auf dem Sand wuchs zunächst ein Ried- oder Úbergangsmoor, später wieder ein Ericales-Sphagnum-Moor auf. Stellenweise ist im Hangenden noch eine geringmächtige Mudde vorhanden, dann folgt die Sandschicht, die das Flöz „C“ vom nächsten Flöz „D“ trennt.

Die Vermoorung setzt im Laufe einer Warmzeit (Zone TA, Tafel IV) ein, deren älteste Abschnitte daher nicht erfaßt sind. Neben den geläufigen thermophilen Gehölzen (Quercus, Ulmus, Carpinus, Corylus, Alnus cf glutinosa) sind wiederum cf Ostrya, Eucommia und Pterocarya vorhanden. Dazu kommt in geringem Maße Pollen weiterer thermophiler Tertiär-Relikte (Clethra-Typ, Phellodendron u. a.). Die thermophile Osmunda ist ebenfalls vorhanden. Trotz des wohl erheblichen Lokaleinflusses kommt der thermophile Charakter der Vegetation klar zum Ausdruck. Anfangs trug das Moor anscheinend den Charakter eines Ried- oder Übergangsmoores (Gramineae, Cyperaceae, Thalictrum, Rubiaceae, Lythrum, Gentianaceae einerseits, Ericales, Sphagnum, Lycopodium of inundatum andererseits), später den eines reinen Ericales-Sphagnum-Moores.

Auf die „Thermophase“ (TA) folgt eine „Kryophase“ (TB), in der die Pollenanteile der thermophilen Taxa ständig abnehmen. Zuerst verschwinden weitgehend die Pollen 
vom Ostrya-Typ, vom Clethra-Typ, von Eucommia und Pterocarya und die Sporen von Osmunda $\left(\mathrm{TB}_{1}\right)$, während der Pollen vom Alnus viridis-Typ erneut langsam zunimmt. Im folgenden Abschnitt ( $\left.\mathrm{TB}_{2}\right)$ kann die deutliche Zunahme der Artemisia-Anteile auf eine gewisse Auflichtung des Waldes hinweisen. Innerhalb des Baumpollens bildet sich eine schwache Betula-Dominanz heraus. Pollen und Sporen "borealer" Elemente, Alnus cf viridis, cf Larix, Lycopodium of selago, werden häufiger, während die Pollenanteile thermophiler Elemente ein Minimum durchlaufen. Trotz der Seeablagerung spielt der Ericales-Pollen immer noch eine große Rolle; vielleicht war die Umgebung mehr oder minder verheidet. Das Klima hatte wohl einen ozeanischen Charakter (Lycopodium cf inundatum, Myrica). Ob es schon als subarktisch bezeichnet werden darf, läßt sich nicht sicher entscheiden. Von der Beantwortung dieser Frage hängt es aber ab, ob der Abschnitt TB als eigene Kaltzeit oder als „Kryophase“ der Tornesch-Warmzeit angesehen wird. Hier wird die zweite Auffassung vertreten. Im Vergleich zum Abschnitt $\mathrm{K}_{0}$ (Tafel II) hatte die Vegetation dort einen deutlicher subarktischen Charakter.

Die jüngere „Thermophase“ der Tornesch-Warmzeit (TC) setzt mit einem Abschnitt ( $\mathrm{TC}_{1}$ ) ein, in dem die Ulmus, cf Ostrya- und Quercus-Anteile wieder etwas zunehmen, während die Alnus of viridis- und cf Larix-Anteile kulminieren. Zunächst wird eine mineralarme Mudde abgelagert, die große Mengen an Pollen und Sporen von Wasserpflanzen führt. Vor allem treten Brasenia-Pollen und Isoetes-Sporen auf, daneben Pollen von Myriophyllum, Nymphaea, Alisma, Utricularia und Potamogeton. Sie weisen auf ein wieder günstigeres Klima hin.

Im folgenden Abschnitt $\left(\mathrm{TC}_{2}\right)$ nehmen die Pollenanteile der „borealen “ Elemente, wie Alnus cf viridis und cf Larix, wieder ab, während die Anteile von Quercus und Ulmus weiter ansteigen. Carpinus- und Eucommia-Pollen sind wieder regelmäßig beteiligt. Im Abschnitt $\mathrm{TC}_{3}$ erscheint dann auch Pterocarya, während Carpinus und Quercus kulminieren.

In der hangenden Mudde (Zone „P“) ist offenbar erneut ein „kalter“ Abschnitt erfaßt worden, von dem sich aber noch nicht sicher sagen läßt, ob er einer Kaltzeit oder einer „Kryophase" angehört.

Die Tornesch-Warmzeit zeigt also bisher eine markante Dreiteilung mit den „Thermophasen " TA und TC und der "Kryophase" TB. Die Pollenflora der "Thermophasen“ ist derjenigen der Nordende-Warmzeit ähnlich, aber anscheinend etwas ärmer an Spuren von Tertiär-Relikten.

\section{Diskussion}

\subsection{Umfang der Lieth-Serie}

Außer dem Hiatus zwischen den Abschnitten $\mathrm{N}_{4}$ und $\mathrm{K}_{0}$ (Tafel II) haben sich bisher keine Hinweise auf wesentliche Schichtlücken ergeben (wenn man von dem gegenüber der Klimabesserung offenbar verspäteten Beginn der Vermoorung in den Flözen „B“ und „C“ absieht). Wahrscheinlich umfaßt aber auch dieser Hiatus keine sehr große Zeitspanne. Die Kalt- und Warmzeiten gehören also einer anscheinend ununterbrochenen Abfolge an. Sie soll als "Lieth-Serie“ bezeichnet werden. Diese Serie setzt sich im Flöz „D“ (Abb. 1) weiter fort und ist auch mit diesem Flöz möglicherweise noch nicht abgeschlossen, doch kann darauf an dieser Stelle nicht eingegangen werden.

\subsection{Kriterien der Lieth-Serie}

Die „warmen" Abschnitte der Lieth-Serie zeichnen sich vor allem durch den Pollenniederschlag von Mischwäldern aus. Unter den Koniferen war hier nur Pinus stärker vertreten (sofern diese überhaupt eine Rolle spielten), während Abies anscheinend fehlte. Picea war wohl durchgehend, aber nur gering verbreitet, möglicherweise in mehreren Arten. Unter den Laubhölzern dominierte Quercus. In den jeweils jüngeren Abschnitten war auch Carpinus stärker vertreten. Regelmäßig waren vor allem Ulmus, cf Ostrya so- 
wie in den jeweils jüngeren Abschnitten Eucommia und Pterocarya beteiligt. Corylus spielte nur eine geringe Rolle. Auffallend ist das nahezu völlige Fehlen von Tilia. Ferner kamen - wenigstens in der weiteren Umgebung - noch einige weitere Tertiär-Relikte vor. Carpinus verhielt sich praktisch wie eine Komponente des „Eichen-Mischwaldes“, so daß eine klare Trennung in "EMW-Zeit“ und „Hainbuchen-Zeit" nicht möglich ist. Die Ericales, darunter Calluna, spielten im jüngeren Teil der Serie eine zunehmend größere Rolle, desgleichen Myrica (die in der Nordende-Warmzeit offenbar noch fehlte) und Lycopodium of inundatum. Daraus kann wohl auf eine zunehmende Ozeanität des Klimas geschlossen werden.

Die „kalten“ Abschnitte zeichneten sich wohl hauptsächlich durch subarktische bis arktische Verhältnisse aus. Mit Ausnahme der Ekholt-Kaltzeit bestand die Vegetation der „kalten“ Abschnitte wohl hauptsächlich aus Ericales-Heiden, durchsetzt mit Kräutern, wie Artemisia, Thalictrum, Lycopodium selago, Selaginella u. a., sowie Gebüschen aus Salix, Myrica, Alnus of viridis und Betula. Auch hieraus läßt sich auf ein ozeanisches Klima schließen.

$\mathrm{Zu}$ Beginn der Warmzeiten und in den Interstadialen hatte die Vegetation einen mehr oder minder „borealen“ Charakter. Sie bestand aus Birken- und Kiefern-Wäldern mit cf Larix und Alnus of viridis, die hier ihre Hauptverbreitung hatten, ferner Myrica und wohl auch mit einem gewissen Anteil thermophiler Gehölze.

\subsection{Stratigraphische Stellung der Lieth-Serie}

Eem- und Holstein-Warmzeit scheiden für die Beurteilung der stratigraphischen Stellung der Lieth-Serie aufgrund ihrer völlig andersartigen Vegetationsentwicklung von vornherein aus.

Der "Cromer-Komplex" ist im einzelnen noch unzureichend bekannt; er scheint heterogen zu sein (ERD 1965, Grüger 1967, West 1962, West \& Wilson 1966). Ein Teil der hierher gestellten Vorkommen enthält Eucommia, ein anderer anscheinend nicht (GRÜGER 1967). Pterocarya und cf Ostrya wurden bisher im "Cromer-Komplex" nicht nachgewiesen, desgleichen Alnus of viridis. Außerdem ist den „cromerzeitlichen " Ablagerungen gemeinsam, daß sich eine EMW-Zeit klar von einer Hainbuchen-Zeit abtrennen läßt (Andersen 1965, Grüger 1967, Müller 1965, West, zit. Benda, Lüttig \& Schneekloth 1967, ZAGWiJn \& Zonneveld 1956). Somit läßt sich die Lieth-Serie nicht in den „CromerKomplex" stellen.

Die typische Pollenvergesellschaftung der Lieth-Serie wurde aber von ZAGwiJn (1957, 1960, 1963) in altpleistozänen Ablagerungen in Holland nachgewiesen, so daß die LiethSerie in diesen Komplex gehört, also älter als der "Cromer-Komplex“, aber jünger als das Pliozän ist.

Im einzelnen ist über das Altpleistozän in Mitteleuropa erst sehr wenig bekannt. Untersuchungen an rein organogenen Ablagerungen lagen bisher noch nicht vor; die Stratigraphie konnte daher praktisch nur an Bohrproben aus tieferen Bohrungen mit z. T. großen Probenabständen erarbeitet werden (ZAGWIJN 1957, 1960, 1963), so daß sich schwer beurteilen läßt, wie weit das Altpleistozän vollständig erfaßt ist.

Der Verlauf der Tornes ch-W a r m z e i t (Tafel IV) läßt sich anscheinend zwanglos mit dem der Wa a l-W a rmzeit (vgl. vor allem Bohrung „Eindhoven I“, ZAGWIJN 1963) vergleichen, wenn man von Lokaleinflüssen absieht. Damit sind die Möglichkeiten einer zwanglosen Konnektierung aber auch schon erschöpft:

Faßt man das Flöz „B“ als ein Groß-Interstadial auf (für das in der holländischen Eburon-Kaltzeit eine Parallele nicht recht erkennbar ist), so sollte die „Nordende-Warmzeit" in das Tegelen gehören und wäre als „Tegelen C“ (ZAGwiJn 1963) anzusehen. Das würde bedeuten, daß das "Tegelen A“ (Belfed-Ton) vom „Tegelen C" durch eine echte Kaltzeit („Ekholt-Kaltzeit“) getrennt sein müßte, wofür aber aus Holland keine Anhaltspunkte vorliegen. Außerdem ist die pollenanalytische Übereinstimmung zwischen dem 
„Tegelen C“ in Holland und der „Nordende-Warmzeit“ im einzelnen nicht gut. In Holland spielen Pinus und Picea eine ungleich größere Rolle als in Lieth, ferner tritt dort Pollen zahlreicher Tertiär-Relikte häufig auf, Carya, Tsuga, Actinidia, Coriaria, Parthenocissus, Magnolia u. a., ferner sind Tilia- und Abies-Pollen in Holland ziemlich regelmäßig beteiligt. Freilich können bei den pollenfloristischen Unterschieden lokale und regionale Vegetationsunterschiede mitspielen.

Sieht man das Flöz „B“ dagegen als eine warmzeitliche Ablagerung an, so müßte diese in das „Tegelen C“, die „Nordende-Warmzeit“ dagegen in das „Tegelen A“ gestellt werden. Man käme in diesem Fall zwar mit der Annahme lediglich eines „kühlen“ Abschnittes zwischen beiden aus, jedoch wäre dann die Übereinstimmung noch schlechter, zumal das „Tegelen A“ in Holland durch die Anwesenheit von Fagus charakterisiert ist.

Weiterhin ist im Flöz „D“ wahrscheinlich noch wenigstens eine weitere Zone mit einer mehr oder minder thermophilen Vegetation (in der Eucommia vorkam) vorhanden, die sich schwer in die holländische Gliederung einfügen läßt.

Die stratigraphische Stellung der Lieth-Serie wird sich daher erst mit größerer Sicherheit beurteilen lassen, wenn der Anschluß an das Pliozän gelingt. Ein laufendes Bohrprogramm des Geologischen Landesamtes Schleswig-Holstein dient u. a. diesem Ziel.

\section{Zitierte Literatur}

Andersen, Sv. Th.: Interglacialer og interstadialer i danmarks kvartaer. - Medd. Dansk Geol. Foren. 15, 4, 486-506, København 1965.

Benda, L., Lüttig, G. \& Schneekloth, H.: Aktuelle Fragen der Biostratigraphie im nordeuropäischen Pleistozän. - Eiszeitalter u. Gegenwart, 17, 218-223, Öhringen 1966.

Dücker, A. \& Menke, B.: Das Altpleistozän von Lieth bei Elmshorn. - Geol. Jb., 86, 791-796, Hannover 1968.

ErD, K.: Pollenanalytische Untersuchungen im Altpleistozän von Voigtstedt in Thüringen. Paläont. Abh. A, II, 2/3, 259-272, Berlin 1965.

GripP, K.: Erdgeschichte von Schleswig-Holstein. 411 S., Neumünster (Wachholtz) 1964.

GrüGER, E.: Vegetationsgeschichtliche Untersuchungen an cromerzeitlichen Ablagerungen im nördlichen Randgebiet der deutschen Mittelgebirge. - Eiszeitalter u. Gegenwart, 18, 204-235, OOhringen 1967.

Lüttig, G.: Menke, B. \& Schneekloth, H.: Über die biostratigraphische Forschung im nordeuropäischen Pleistozän-Stand 1967. - Eiszeitalter u. Gegenwart, 18, 236-239, Öhringen 1967.

MüLLER, H.: Eine pollenanalytische Neubearbeitung des Interglazial-Profils von Bilshausen (Unter-Eichsfeld). - Geol. Jb., 83, 327-352, Hannover 1965.

Oberdorfer, E.: Pflanzensoziologische Exkursionsflora für Süddeutschland. 987 S., Stuttgart (Ulmer) 1962.

WANG, C. W.: The forests of China with a survey of grassland and desert vegetation. - Maria Moors Cabot Foundation publ. 5, Cambridge, Mass. 1961.

West, R.: Vegetational history of the Early Pleistocene of the Royal Society borehole at Ludham, Norfolk. - Proc. Roy. Soc. B, 155, 437-453, London 1962.

West, R. \& Wilson, D. G.: Cromer Forest Bed Series. - Nature 209, 5022, 497-498, London 1966.

Zagwijn, W. H.: Vegetation, climate and time correlations in the Early Pleistocene of Europe. Geol. en Mijnb., N. S. 19, 233-244, s'Gravenhage 1957.

- : Aspects of the Pliocene and Early Pleistocene vegetation in the Netherlands. - Meded. Geol. Sticht., Serie C-III-1 No. 5, 5-78, Maastricht 1960.

- : Pollen-analytic investigations in the Tiglian of the Netherlands. - Meded. Geol. Sticht. N. S., 16, 49-72, Maastricht 1963.

Zagwisn, W. H. \& Zonneveld, J. I. S.: The interglacial of Westerhoven. - Geol. en Mijnb. N. S., 18, 37-46, s'Gravenhage 1956.

Manuskr, eingeg. 24. 12. 1968.

Anschrift des Verf.: Dr. B. Menke, Geologisches Landesamt Schleswig-Holstein, 23 Kiel, Mecklenburger Straße 22/24. 